\title{
SUSTAINABLE PRODUCT PORTFOLIO AND PROCESS DESIGN OF THE FOREST BIOREFINERY
}

\author{
Matty Janssen \& Paul Stuart \\ Department of Chemical Engineering, École Polytechnique de Montral, Quebec Canada \\ paul.stuart@polymtl.ca
}

\section{INTRODUCTION}

The North American forestry industry has been challenged in recent years by high energy costs, increasing fibre costs, and aging mill assets that have not been adequately modernized [1]. The so-called forest biorefinery (FBR) has emerged as an interesting candidate for reviving the industry. The FBR may use ligno-cellulosic (woody, agricultural) biomass as raw material and implies full utilization of biomass for the production of fibres, chemicals and/or energy, and thus offers an alternative strategy for its utilization. Its design should consider both the retrofit design of pulp and paper mill processes and the product portfolio [2]. There is an apparent complexity in this development, and therefore a rigorous design framework is needed.

The concept of sustainability consists of three dimensions: economic, environmental and social. However, design typically emphasizes economic objectives for decision making between process alternatives, and the environmental and social aspects of alternatives are considered as constraints (e.g. [3. 4]). Multi-criteria decision making (MCDM) methods may be used to consider all sustainability dimensions as objectives.

\section{METHODOLOGY}

\section{Design framework}

Figure 1 shows a design framework that takes into account the design of the product portfolio and biorefinery production process [7]. It consists of a set of process systems engineering (PSE) tools that together cover all aspects of FBR process and portfolio design. In this framework, an MCDM method is used for design decision making based on the analysis results using the PSE tools. MCDM methods raise the awareness of the decision maker(s) about the problem complexity and they can be used to address decision uncertainty.

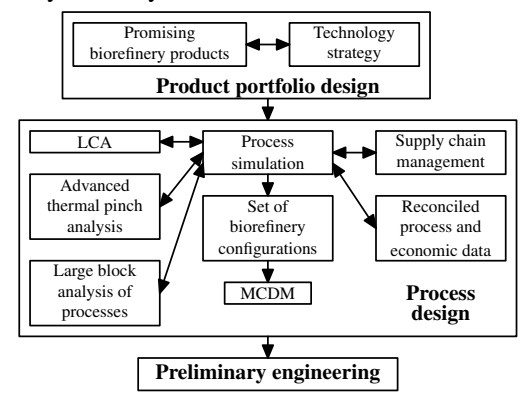

Figure 1: Process systems engineering framework for forest biorefinery design

\section{Panel-based MCDM}

An expert panel was carried out in order to establish the importance by weighting the selected criteria. The MCDM methodology consists of a prepanel and a panel phase. During the pre-panel phase, the objective, the decision criteria and the decision structure are defined, and the decision problem and weighting procedure introduced to the panel. The first step of an MCDM panel phase involves the discussion of the drivers and barriers by the panel. This discussion is critical in order to raise the awareness of the decision maker(s) about the criteria complexity and implications, and as a consequence the panel is better equipped to address decision uncertainty by understanding the different panel member perspectives. Finally, using a weighting method, the relative importance is given to each of the criteria.

This methodology was applied to determine the importance of a set of drivers and barriers for biorefinery implementation using Analytic Hierarchy Process [5]. In another study it was applied to determine the importance of decision criteria involved in very-early process design selection in the context of second generation ethanol production at a pulp and paper mill. The weighting method used in this study was based on Multi-Attribute Utility Theory [6], i.e. a trade-off method.

\section{RESULTS \& DISCUSSION}

\section{Drivers and barriers for biorefinery implementation $[\overline{8}]$}

Calculation of the driver weights revealed that the drivers directly related to the economic health of the company are the most important (Drivers I and IV, Figure 2a). It also revealed that the short-term economic health (Driver I) is more important than the long-term health (Driver IV). The panel members understood that long-term strategy is essential, however less important than short-term gains. The forestry industrys risk-averse investment culture was found to be the main barrier for implementation of the biorefinery (Figure2 2 ). Furthermore, the policy-related barrier (Barrier I) was least important, yet its weight was significantly higher than the weight for the policy-related driver (Driver III). Although the industry wants to minimize its reliance on government policy, these policies need to be known before the industry embarks on the biorefinery.

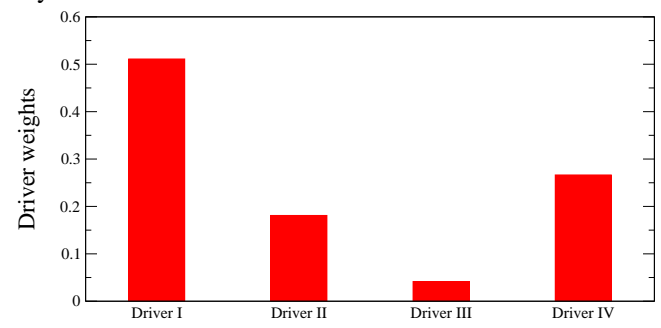

Driver I: Commodity pulp and paper product provide insufficient margins for remaining Driver II: Future access of raw materials at current prices for the core business may be better guaranteed through biorefinery implementation (n) change and other issues for biorefinery implementation support

Driver IV:The current business model of North American forestry companies will not increase

a. the value of the company, and is no longer acceptable to the stock market

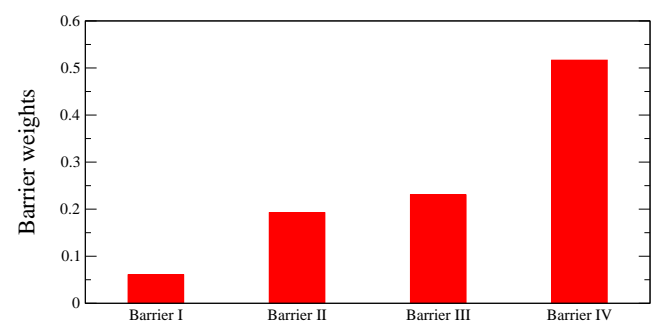

Barrier I: There is uncertainty about future government policy with regards to biorefinery

implementation

Barrier II: Early implementers may incur high risk due to inefficient early-generation technologies and be unaware of new emerging biorefinery technologies that are more efficient critical for success in most cases, and is difficult to secure at this early stage Barrier IV:The industry's risk-averse culture favors short-term investment, and may cond

b. Barrier IV:The industry's risk-averse culture favors short-term investment, and may compromise

Figure 2: Weights assigned to a) drivers and b) barriers for the implementation of the biorefinery

\section{Very-early stage design decision making [9]}

The adjusted ROI was the most important criterion according to the panel members (Figure 3 and reflected the importance of having more credible data for profitability evaluation. Furthermore, the energy integration potential was important which underlines the need for energy management. This will lead to lower production cost of the core pulp and paper products. The environmental criterion had the lowest consensus among the panel members, underlining it may need a clearer definition as a "show stopper".

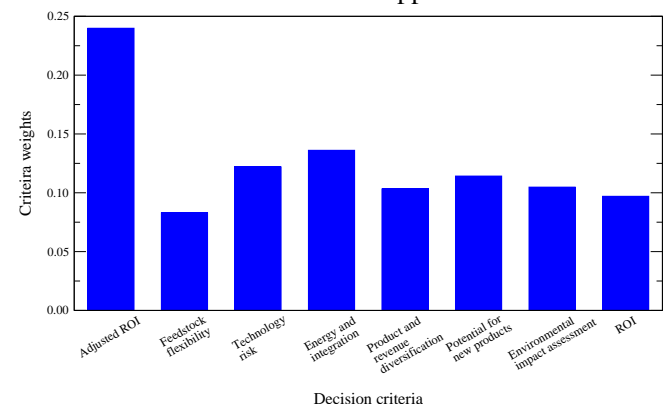

Figure 3: Weighting of criteria for very-early stage design decision making

\section{REFERENCES}

[1] P. Stuart, Pulp Pap-Canada, 2006;107(6):13-6

[2] V. Chambost et al, Pulp Pap-Canada, 2008;109(7-8):19-27

[3] M. Janssen, et al, Pulp Pap-Canada, 2006;107(9):33-7

[4] E. Uerdingen, et al, AIChE J, 2003;49(9):2400-2418

[5] T.L. Saaty, McGraw-Hill, 1980

[6] R. Keeney and H. Raiffa, Cambridge University Press, 1976

[7] M. Janssen et al, $8^{\text {th }}$ World Congress of Chemical Engineering, 2009

[8] M. Janssen and P. Stuart, Pulp Pap-Canada, 2010

[9] J. Cohen et al, Pulp Pap-Canada, 2010 